ISSN-L 2077-0014

DOI: https://doi.org/10.33326/26176068.2021.3.1157

Artículo original/Original article/Artigo original

\title{
Factores sociodemográficos y nivel de conocimiento asociado al uso de la anticoncepción oral de emergencia
}

Sociodemographic factors and level of knowledge associated with the use of emergency contraception pills Fatores sociodemográficos e nível de conhecimento associados ao uso de anticoncepção de emergência

\author{
María Angela Velarde Cárdenas ${ }^{1 a, d, g}$ \\ Elena Cachicatari Vargas ${ }^{1 \mathrm{~b}, \mathrm{e}, \mathrm{f}, \mathrm{h}}$ \\ Natanael Luis Imaña Morales ${ }^{c}$ \\ Katty Giovana Mendoza Mamania,d,g
}

(D) https://orcid.org/0000-0002-7221-8418

(iD) https://orcid.org/0000-0002-9843-432X

(D) https://orcid.org/0000-0002-5516-9386

(D) https://orcid.org/0000-0001-8821-8079

\begin{abstract}
Resumen
Objetivo: Determinar los factores sociodemográficos y nivel conocimiento asociado al uso de la anticoncepción oral de emergencia en usuarias del centro médico INPPARES de la ciudad de Tacna, en el año 2020. Material y métodos: El estudio fue básico de nivel correlacional, no probabilístico con un muestreo por conveniencia de 1200 mujeres atendidas en el centro médico, quienes fueron encuestadas para medir su nivel de conocimiento y uso de la anticoncepción oral de emergencia (AOE), identificando factores sociodemográficos como la edad, estado civil y grado de instrucción. Para el análisis de datos se utilizó el software SPSS 25 y se empleó Chi cuadrado con un nivel de significancia de $95 \%$. Resultados: Se identificó que el $57,5 \%$ de las mujeres encuestadas tienen un conocimiento bajo de la anticoncepción oral de emergencia frente a un $7,6 \%$ con un conocimiento alto. Con respecto al uso del AOE se identificó que 85,9\% lo utilizó por lo menos una vez en su vida; el 47,4 \% lo usó por lo menos una vez al año; el 50,6 \% tienen entre 15 y 25; el 59,1 \% cuenta con grado de instrucción de nivel secundario; y el estado civil del 64,9 \% es soltera. Conclusión: Existe relación entre el nivel de conocimientos y factores sociodemográficos asociados al uso de la anticoncepción oral de emergencia.
\end{abstract}

Palabras clave: anticoncepción oral emergencia, nivel conocimiento, planificación familiar

\begin{abstract}
Objective: To determine the sociodemographic factors and level of knowledge associated with the use of emergency oral contraception in users of the INPPARES medical center in the city of Tacna, in 2020. Material and methods: The study was basic at a correlational level, not probabilistic with a sampling for convenience of 1200 women attended in the medical center, who were surveyed to measure their level of knowledge and use of emergency contraception (EC), identifying sociodemographic factors such as age, marital status and level of education. SPSS 25 software was used for data analysis and Chi-square was used with a significance level of $95 \%$. Results: It was identified that $57.5 \%$ of the surveyed women have a low knowledge of emergency oral contraception compared to $7.6 \%$ with a high knowledge. Regarding the use of the EC, it was identified that $85.9 \%$ used it at least once in their life; $47.4 \%$ used it at least once a year; $50.6 \%$ are between 15 and 25; $59.1 \%$ have a secondary level education degree; and the marital status of $64.9 \%$ is single. Conclusion: There is a relationship between the level of knowledge and sociodemographic factors associated with the use of emergency contraception.
\end{abstract}

Keywords: emergency contraception, family planning, knowledge level

\footnotetext{
1 Universidad Nacional Jorge Basadre Grohmann. Tacna, Perú

a Lic. en Obstetricia

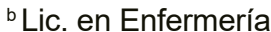

${ }^{c}$ Cirujano dentista

¿ Magister en Salud Pública

e Magister en Tecnología Educativa

${ }^{f}$ Dra. en Ciencias: Enfermería

g Docente de la Escuela de Obstetricia

${ }^{\mathrm{h}}$ Docente de la Escuela de Enfermería
} 


\begin{abstract}
Resumo
Objetivo: Determinar os fatores sociodemográficos e nível conhecimento associado ao uso do anticoncepção de emergência em usuárias do centro médico INPPARES da cidade de Tacna, 2020. Material e métodos: O estudo foi básico de nível correlacional, não probabilístico com uma amostragem por conveniência de 1200 mulheres atendidas no centro médico, que foram entrevistadas para medir seu nível de conhecimento e uso de anticoncepção de emergência (AE) identificando factores sociodemográficos como a idade, estado civil e grau de instrução; para a análise de dados utilizou-se o software SPSS 25 e utilizou-se Qui-quadrado com um nível de significância de 95 \%. Resultados: Foi identificado que 57,5 \% das mulheres entrevistadas têm um conhecimento deficiente da anticoncepção de emergência, contra $7,6 \%$ com bons conhecimentos. No que diz respeito à utilização do AE, foi identificado que $85,9 \%$ o utilizou pelo menos uma vez na sua vida; 47,4 \% o utilizou pelo menos uma vez por ano; 50,6 \% têm entre 15 e 25; 59,1 \% têm um grau de instrução de nível secundário; e o estado civil de 64,9 \% é solteiro. Conclusão: Existe uma relação entre o nível de conhecimentos e os factores sociodemográficos associados ao uso do anticoncepção de emergência.
\end{abstract}

Palavras-chave: anticoncepção de emergência, nível de conhecimento, planejamento familiar

\section{Introducción}

La planificación familiar es uno de los temas y herramientas utilizadas para prevenir un embarazo no deseado y así, en el futuro, poder evitar muertes maternas, este es un tema muy importante en la salud pública.

En el año 1948, la Organización Mundial de la Salud (OMS) definió a la salud como un bienestar no solamente físico sino mental y social, que no necesariamente signifique la ausencia de una enfermedad. En tal sentido la planificación familiar en nuestros tiempos, específicamente la utilización de métodos anticonceptivos se ha vuelto fundamental en la etapa reproductiva de las personas. En muchas de sus publicaciones manifiesta el incremento de los embarazos no deseados, siendo la población más afectada los adolescentes, en muchos de los casos, debido a la falta de información o acceso a métodos anticonceptivos incluyendo la anticoncepción oral de emergencia. ${ }^{1}$

Según la OMS, entre el año 2010 y 2014, a nivel mundial, se promedió al año 56 millones de abortos, posiblemente por el mal uso de los métodos anticonceptivos. $^{2}$

El incremento de tasa de embarazos no deseados o no planificados existentes en nuestro país y a nivel internacional hace que la problemática de salud reproductiva planteada en la presente investigación sea de importancia, estos pudieron ser prevenidos, como está ya reconocido, por el uso de anticonceptivos de emergencia. Se atribuye la causa primordialmente al deficiente conocimiento y aceptación a esta alternativa anticonceptiva. ${ }^{3}$

En el Perú, según la UNFPA, la mitad de los niños recién nacidos no fueron planificados, convirtiendo muchos de ellos en embarazos no deseados, contabilizando un tercio de las usuarias de planificación familiar con dependencia de uso de los métodos por parte de sus parejas. ${ }^{3}$
La anticoncepción oral de emergencia (AOE) es una píldora muy utilizada en nuestro país y puede ser obtenida en cualquier botica o farmacia sin necesidad de una receta médica, sin previa orientación sobre su uso correcto. ${ }^{4}$ Según un estudio de la Revista Peruana de Medicina Experimental y Salud Pública, en el 2009, las mujeres acceden a su venta de forma privada, también en este estudio se reportó que dos de cada tres mujeres estudiadas habían usado la AOE al menos una vez en su vida. ${ }^{5}$

La AOE muchas veces es usada no solo en caso de emergencia por las parejas, sino como un método regular de protección y es lo que cotidianamente manifiestan las usuarias en las consultas. Este método de emergencia hormonal, compuesto por levonorgestrel o acetato de ulipristal, se ha convertido en una herramienta para poder evitar embarazos, cuyos mecanismos de acción, en la mayoría de los casos, consiste en el espesamiento del moco cervical y en la reducción de la motilidad de la trompa de Falopio.

Actualmente, en muchos establecimientos de salud se encuentran disponibles métodos anticonceptivos que se pueden adquirir gratuitamente incluyendo la anticoncepción oral de emergencia, pero en su mayoría como método de Yuzpe.

El método de Yuzpe, en el Ministerio de Salud, consiste en la toma de cuatro tabletas de anticonceptivo oral combinado (AOC), luego de doce horas se repite la dosis, siendo esta la técnica más accesible para las usuarias que no cuentan con suficientes ingresos económicos y que acuden a los establecimientos de salud, siendo su tasa de falla dos de cada cien mujeres, teniendo una eficacia del $75 \%$, según la Revista Cubana de Obstetricia y Ginecología. ${ }^{6}$

Por tal motivo, es importante medir el nivel de conocimiento de las personas, especialmente las mujeres, porque la mayoría de los métodos son usados por ellas. En el caso de la AOE, es necesario 
que sepan cuántas veces pueden tomarla en un año y los posibles efectos secundarios que podría causar su mal uso. Además, de los diferentes factores sociodemográficos que podrían intervenir y las barreras que impidan el adecuado uso de este método.

Es importante que, en el Instituto de Paternidad Responsable (INPPARES), siendo una de las instituciones líderes en la salud sexual y reproductiva, donde acude una población multicultural, se pueda investigar el nivel de conocimiento de las usuarias.

Por consiguiente, en el presente estudio se formula la pregunta: ¿En realidad, las usuarias que acuden a un servicio de salud sexual y reproductiva conocen sobre el AOE y su uso correcto?

Como se puede identificar en el presente estudio, el mal uso de anticonceptivos podría desencadenar un embarazo no deseado. La anticoncepción oral de emergencia muchas veces es mal usada ya sea por la mala administración en tiempo, mala administración de dosis o su uso excesivo. Teniendo en cuenta que solo se usa en casos de emergencia para posteriormente cuidarse con un método anticonceptivo, ya sea de corto o largo plazo, más seguro.

Existen muchas causas relacionadas al mal uso de la AOE como la falta de conocimiento y efectos secundarios, no buscar fuentes que ofrezcan información científica confiable, la limitación de comunicación con sus padres, por sus creencias religiosas, el acceso a métodos anticonceptivos de salud; ya sea en el caso de adolescentes, por vergüenza o miedo a ser juzgada o por el tiempo de espera.

Las mujeres, que a partir de ahora las llamaremos usuarias, acuden a establecimientos de salud, ya sean hospitales, clínicas o centros de salud para poder usar algún método anticonceptivo o recibir algún tipo de orientación o consejería acerca del tema.

Asimismo, muchas de estas usuarias acuden al centro médico INPPARES en la ciudad de Tacna, centro especialista en temas de salud sexual y reproductiva desde hace 43 años, cuya misión es "contribuir al bienestar sexual y reproductivo" de las personas, en muchos casos brindado consejería en salud sexual y reproductiva de forma gratuita por parte de profesionales capacitados y actualizados en el tema.

El objetivo del presente estudio fue determinar los factores sociodemográficos y nivel conocimiento asociado al uso de la anticoncepción oral de emergencia en usuarias del centro médico INPPARES de la ciudad de Tacna, 2020; con el propósito de reducir los embarazos no deseados, además de aumentar el nivel de conocimientos de las usuarias que acuden a establecimientos de salud, en especial a los servicios de salud sexual y reproductiva.

\section{Material y métodos}

El presente estudio fue descriptivo, no experimental porque no se manipularon las variables, ya que son solo analizadas; y transversal porque se hizo en un determinado tiempo.

El nivel de investigación es de tipo correlacional para así poder medir el grado de relación de las variables en una misma persona, poder determinar si se correlacionan y en qué grado e intensidad.

La muestra estuvo compuesta por 1200 mujeres en edad fértil atendidas en el Servicio de Salud Sexual y Reproductiva en centro médico INPPARES Tacna. Para seleccionar la muestra se optó por emplear el método de muestreo no probabilístico por conveniencia, obteniendo las muestras de acuerdo a la facilidad de acceso a las usuarias.

Se incluyó a las usuarias atendidas en el Servicio de Salud Sexual y Reproductiva del centro médico INPPARES que actualmente viven en la ciudad de Tacna y a quienes llenen adecuadamente los cuestionarios brindados. Se excluyó a usuarias atendidas en otros servicios médicos. No se consideró a mujeres que vivan en otra ciudad y solo sean transeúntes en el servicio, por lo tanto, el grupo seleccionado es el que mejor se ajusta a los requerimientos del objetivo de la investigación.

Como variable independiente se consideró a los factores sociodemográficos y el nivel de conocimiento sobre la anticoncepción oral de emergencia, y la variable dependiente al uso de la anticoncepción oral de emergencia.

Como instrumento se utilizó un cuestionario validado por expertos en el tema, con preguntas dicotómicas y múltiples relacionadas a factores sociodemográficos y nivel de conocimiento asociado al uso de la anticoncepción oral de emergencia, previo consentimiento informado de las usuarias atendidas en el Servicio de Salud Sexual y Reproductiva.

Camacaro afirmó que las técnicas de recolección de datos son instrumentos de recolección de datos, es decir, cualquier recurso de que pueda valerse el investigador para acercarse a los fenómenos y extraer de ellos la información. ${ }^{7}$

Como consideraciones éticas se tomó en cuenta la Declaración de Helsinki de la Asociación Médica Mundial sobre aspectos éticos internacionales 
para los estudios en seres humanos. ${ }^{8}$

Para el procesamiento de la información se utilizó el software estadístico SPSS versión 25. Prueba estadística de correlación de Chi cuadrado. Los resultados se presentaron en tablas descriptivas con frecuencias absolutas y relativas.

\section{Resultados}

En la tabla 1 se observa la muestra conformada por 1200 usuarias, en donde 549 de ellas $(45,8 \%)$ tienen entre 15-25 años; 351 usuarias (29,3\%), entre 26-35 años; 95 usuarias (7,9\%), entre 36-45 años; y 36 usuarias (3\%), entre 46 a más. De todas las mujeres participantes en este estudio, hemos encontrado que el 85,9 \% usaron anticoncepción oral de emergencia.

\section{Tabla 1}

Edad de las usuarias atendidas en INPPARES asociada al uso de la $A O E$

\begin{tabular}{|c|c|c|c|c|c|c|}
\hline \multirow{3}{*}{ Edad } & \multicolumn{4}{|c|}{ Uso de la AOE } & \multirow{2}{*}{\multicolumn{2}{|c|}{ Total }} \\
\hline & \multicolumn{2}{|c|}{ Sí } & \multicolumn{2}{|c|}{ No } & & \\
\hline & $\mathrm{N} .^{\circ}$ & $\%$ & $\mathrm{~N} .^{\circ}$ & $\%$ & $\mathrm{~N} .^{\circ}$ & $\%$ \\
\hline De 15 a 25 años & 549 & 45,8 & 58 & 4,8 & 607 & 50,6 \\
\hline De 26 a 35 años & 351 & 29,3 & 41 & 3,4 & 392 & 32,7 \\
\hline De 36 a 45 años & 95 & 7,9 & 35 & 2,9 & 130 & 10,8 \\
\hline De 46 a más & 36 & 3 & 35 & 2,9 & 71 & 5,9 \\
\hline Total & 1031 & 85,9 & 169 & 14,1 & 1200 & 100 \\
\hline$X 2=105,0$ & & GL & & $P=$ &, 000 & 0,05 \\
\hline
\end{tabular}

En la Tabla 2 se observa la muestra de 1200 usuarias, de las cuales, 702 usuarias, que representan el $58,5 \%$ son solteras y usaron la anticoncepción oral de emergencia; seguido de 179 usuarias, representadas por el 14,9\% con estado civil conviviente, que utilizaron la anticoncepción oral de emergencia; 147 usuarias (12,3\%) tienen un estado civil casada, solo 3 usuarias viudas $(0,3 \%)$ refieren haber utilizado la $\mathrm{AOE}$.

\section{Tabla 2}

Estado civil de las usuarias atendidas en INPPARES asociado al uso de la AOE

\begin{tabular}{|c|c|c|c|c|c|c|}
\hline \multirow{3}{*}{ Estado civil } & \multicolumn{4}{|c|}{ Uso de la AOE } & \multirow{2}{*}{\multicolumn{2}{|c|}{ Total }} \\
\hline & \multicolumn{2}{|c|}{ Sí } & \multicolumn{2}{|c|}{ No } & & \\
\hline & $\mathrm{N} .^{\circ}$ & $\%$ & $\mathrm{~N} .^{\circ}$ & $\%$ & $\mathrm{~N} .^{\circ}$ & $\%$ \\
\hline Soltera & 702 & 58,5 & 77 & 6,4 & 779 & 64,9 \\
\hline Casada & 147 & 12,3 & 59 & 4,9 & 206 & 17,2 \\
\hline Conviviente & 179 & 14,9 & 30 & 2,5 & 209 & 17,4 \\
\hline Viuda & 3 & 0,3 & 3 & 0,3 & 6 & 0,5 \\
\hline Total & 1031 & 85,9 & 169 & 14,1 & 1200 & 100 \\
\hline \multicolumn{2}{|c|}{$X 2=53,839$} & \multicolumn{2}{|c|}{$G L=3$} & \multicolumn{3}{|c|}{$P=0,000<0,05$} \\
\hline
\end{tabular}

En la Tabla 3 se observa la muestra de 1200 usuarias, de las cuales 604 presentan un grado de instrucción de nivel secundario, siendo el 50,3\% usuarias que utilizaron la AOE; 352 usuarias cuentan con un grado de instrucción universitario, obteniendo un $29,3 \%$ que refieren haber utilizado la AOE; 55 usuarias $(4,6 \%)$ con grado de instrucción superior no universitario utilizaron la AOE; finalmente, solo 20 usuarias $(1,7 \%)$ cuenta con un grado de instrucción primaria.

\section{Tabla 3}

Grado de instrucción de las usuarias atendidas en INPPARES asociado al uso de la AOE

\begin{tabular}{|c|c|c|c|c|c|c|}
\hline \multirow{3}{*}{$\begin{array}{l}\text { Grado de } \\
\text { instrucción }\end{array}$} & \multicolumn{4}{|c|}{ Uso de la AOE } & \multirow{2}{*}{\multicolumn{2}{|c|}{ Total }} \\
\hline & \multicolumn{2}{|c|}{ Sí } & \multicolumn{2}{|c|}{ No } & & \\
\hline & $\mathrm{N} .^{\circ}$ & $\%$ & $\mathrm{~N} .^{\circ}$ & $\%$ & $\mathrm{~N} .^{\circ}$ & $\%$ \\
\hline Primaria & 20 & 1,7 & 7 & 0,6 & 27 & 2,3 \\
\hline Secundaria & 604 & 50,3 & 105 & 8,8 & 709 & 59,1 \\
\hline $\begin{array}{l}\text { Superior } \\
\text { universitaria }\end{array}$ & 352 & 29,3 & 47 & 3,9 & 399 & 33,3 \\
\hline $\begin{array}{l}\text { Superior no } \\
\text { universitaria }\end{array}$ & 55 & 4,6 & 10 & 0,8 & 65 & 5,4 \\
\hline Total & 1031 & 85,9 & 169 & 14,1 & 1200 & 100 \\
\hline \multicolumn{2}{|c|}{$X 2=5,580$} & \multicolumn{2}{|c|}{$\mathrm{GL}=3$} & \multicolumn{3}{|c|}{$P=0,152<0,05$} \\
\hline
\end{tabular}

En la Tabla 4 se observa la muestra de 1200 usuarias, donde el $49,8 \%$ de usuarias que utilizaron la $A O E$ tienen un nivel de conocimiento bajo, frente al $29,3 \%$ de usuarias con conocimiento medio del AOE, solo el 6,9 \% mostró un nivel de conocimiento alto, siendo 83 usuarias quienes usaron adecuadamente la AOE.

\section{Tabla 4}

Nivel de conocimiento de las usuarias atendidas en INPPARES asociado al uso de la AOE

\begin{tabular}{|c|c|c|c|c|c|c|c|c|}
\hline \multirow{3}{*}{$\begin{array}{l}\text { Estado } \\
\text { civil }\end{array}$} & \multicolumn{6}{|c|}{ Uso de la AOE } & \multirow{2}{*}{\multicolumn{2}{|c|}{ Total }} \\
\hline & \multicolumn{2}{|c|}{ Alto } & \multicolumn{2}{|c|}{ Medio } & \multicolumn{2}{|c|}{ Bajo } & & \\
\hline & $\mathrm{N} .^{\circ}$ & $\%$ & $\mathrm{~N} .^{\circ}$ & $\%$ & $\mathrm{~N} .^{\circ}$ & $\%$ & $\mathrm{~N} .^{\circ}$ & $\%$ \\
\hline Sí & 83 & 6,9 & 351 & 29,3 & 597 & 49,8 & 1031 & 85,9 \\
\hline No & 8 & 0,7 & 68 & 5,7 & 93 & 7,8 & 169 & 14,1 \\
\hline Total & 91 & 7,6 & 419 & 34,9 & 690 & 57,5 & 1200 & 100 \\
\hline \multicolumn{3}{|c|}{$X 2=3,909$} & & \multicolumn{2}{|c|}{$\mathrm{GL}=2$} & \multicolumn{3}{|c|}{$P=0,142<0,05$} \\
\hline
\end{tabular}


En la Tabla 5 se observa la muestra de 1200 usuarias, de las cuales, un $26,3 \%$ de usuarias, entre 15-25 años, utilizaron la AOE dos veces al año, frente a un $22,6 \%$ (271 usuarias) entre los
26-35 años que lo utilizaron solo una vez en el año y solo un $4,6 \%$ (55 usuarias) lo utilizaron de 3 veces a más en un año, con edades entre los 15-25 años.

\section{Tabla 5}

Edad de las usuarias atendidas en INPPARES asociada a la frecuencia de uso de la AOE

\begin{tabular}{|c|c|c|c|c|c|c|c|c|c|c|}
\hline \multirow{3}{*}{ Edad } & \multicolumn{8}{|c|}{ Frecuencia uso de la $\mathrm{AOE}$} & \multirow{2}{*}{\multicolumn{2}{|c|}{ Total }} \\
\hline & \multicolumn{2}{|c|}{1 ves al año } & \multicolumn{2}{|c|}{2 veces al año } & \multicolumn{2}{|c|}{$\begin{array}{c}3 \text { veces o más en } \\
\text { el año }\end{array}$} & \multicolumn{2}{|c|}{ Nunca } & & \\
\hline & $\mathrm{N}^{\circ}{ }^{\circ}$ & $\%$ & $\mathrm{~N}^{\circ}{ }^{\circ}$ & $\%$ & $\mathrm{~N} .^{\circ}$ & $\%$ & $\mathrm{~N} .^{\circ}$ & $\%$ & $\mathrm{~N} .^{\circ}$ & $\%$ \\
\hline De 15 a 25 años & 179 & 14,9 & 315 & 26,3 & 55 & 4,6 & 58 & 4,8 & 607 & 50,6 \\
\hline De 26 a 35 años & 271 & 22,6 & 54 & 4,5 & 26 & 2,2 & 41 & 3,4 & 392 & 32,7 \\
\hline De 36 a 45 años & 84 & 7 & 10 & 0,8 & 1 & 0,1 & 35 & 2,9 & 130 & 10,8 \\
\hline De 46 años & 35 & 2,9 & 1 & 0,1 & 0 & 0 & 35 & 2,9 & 71 & 5,9 \\
\hline \multirow[t]{2}{*}{ Total } & 569 & 47,4 & 380 & 31,7 & 82 & 6,8 & 169 & 14,1 & 1200 & 100 \\
\hline & \multicolumn{2}{|c|}{$X 2=397,873$} & \multicolumn{2}{|c|}{$\mathrm{GL}=9$} & \multicolumn{4}{|c|}{$P=0,000<0,05$} & & \\
\hline
\end{tabular}

\section{Discusión}

De la totalidad de mujeres participantes en este estudio, se encontró un elevado porcentaje de uso de anticoncepción oral de emergencia (85,9\%), en algún momento.

Las edades que más utilizaron la $\mathrm{AOE}$ estuvieron entre los $15-25$ años con un $45,8 \%$, muy similar al estudio de Cardenas García L. con una población de entre 19-21 años con un $27 \%$ usó la AOE. ${ }^{9}$ Mientras que Nathaly Reyes S. refiere que las usuarias entre 16 y 25 años que viven en Lima utilizaron la AOE en un 60,8 \%. ${ }^{4}$ Por otro lado, Flores Pardave R. obtuvo como resultado que las usuarias que acuden al Hospital Materno Infantil Carlos Showing Ferrari mostraron edades entre 18-40 años (no se estudió el uso). ${ }^{10}$

En cuanto al estado civil, se obtuvo como resultado que un $58,5 \%$ quienes sí utilizaron la AOE son solteras, a diferencia de Flores Pardave con una población con estado civil conviviente $(54,3 \%)$ posiblemente por la edad de las usuarias atendidas. ${ }^{10}$ En cambio, Nathaly Reyes obtuvo un $86 \%$ de usuarias solteras ya que las edades predominantes son entre 16 y 25 años, muy similar a las del presente estudio. ${ }^{4}$

En el caso de grado de instrucción, el más común en la muestra es el de secundaria con un $50,3 \%$. Es complicado buscar contraste con esta subvariable ya que las anteriores investigaciones solo se enfocaron en un grupo etario específico.

Respecto al nivel de conocimientos asociado al uso de la $A O E$, se obtuvo como resultado que el $49,8 \%$ que sí utilizaron la AOE tienen un nivel de conocimiento bajo, a diferencia de Estrella Cóndor, donde el nivel de conocimiento fue de logro previsto $(37,77 \%)$ en relación al uso $(36,17 \%)$ fue adecuado; sobre los estudiantes que sí usaron el anticonceptivo oral de emergencia. ${ }^{11}$ Santander Mamani refiere que el nivel de conocimiento sobre el uso de la píldora anticonceptiva de emergencia es mayormente regular con 46,1\%, seguido de deficiente con 36,8 $\%$ de adolescentes que utilizaron la AOE. ${ }^{12}$ Mientras que Villena encontró que del total de la población usuaria predomina el conocimiento bajo sobre anticoncepción de emergencia hormonal, siendo la población adolescente la entrevistada. ${ }^{13}$ Entonces, podemos concluir que la población, en especial la adolescente, probablemente no posee la información completa sobre esta anticoncepción, lo que pone en riesgo su salud sexual y reproductiva. Por lo tanto, nuestro estudio aplicado en mujeres, demuestra que la gran mayoría tienen nivel secundario, mientras que en los estudios donde se observa nivel de conocimiento alto fue en universitarias de la población investigada. 
Con respecto a cuántas veces en el año usan la AOE, se obtuvo como resultado $47,4 \%$, siendo las edades más predominantes de 26 a 35 años que lo utilizaron por lo menos una vez, frente a un $31,7 \%$ que lo utilizaron dos veces, es complicado buscar contraste con esta subvariable ya que las anteriores investigaciones solo se enfocaron en un grupo etario específico.

\section{Referencias}

1. World Health Organization. Constitución [Internet] Ginebra: Organización Mundial de la Salud; 1985 [consultado 2020 abril 05]. Disponible en: https://apps.who.int/iris/ bitstream/handle/10665/36853/9243602519. pdf?sequence $=1$

2. Organización Mundial de la Salud. Prevención del aborto peligroso [Internet] Who.int. [consultado 2019 marzo 23]. Disponible en: https://www.who.int/es/news-room/fact-sheets/ detail/preventing-unsafe-abortion

3. Fondo de Población de las Naciones Unidas. [Internet] Perú: Unfpa; 2019 [consultado 2020 marzo 23]. Disponible en: https://peru.unfpa. org/es $/$ news $/ \mathrm{m} \% \mathrm{C} 3 \% \mathrm{~A} 1 \mathrm{~s}$-de-la-mitad-de-lasmujeres-peruanas-embarazan-sin-planificarlo

4. Reyes-Serrano N, Morales-Alvarado S, Valdivieso-Olivia V. Factores asociados al uso de la anticoncepción oral de emergencia por estudiantes de Obstetricia de una universidad privada de Lima, Perú, 2016. Revista de investigación [Internet] 2016 [consultado 2019 marzo 10]; (6): 49. Disponible en: https://www. who.int/governance/eb/who_constitution_ sp.pdf

5. Quispe-Pineda D, Rebolledo-Ponietsky K, Ganoza-CaleroAM, Miranda-Medina J, ElejaldeFarfán N, Arévalo-Revelo D, et al. Increased use of oral emergency contraception in Peru: Reconsidering free access to it. Rev Peru Med Exp Salud Publica. 2016;33(4):839-40.

6. Peláez-Mendoza J. El uso de métodos anticonceptivos en la adolescencia. Revista Cubana de Obstetricia y Ginecología [Internet] 2016 Mar [consultado 2021 marzo 23]; 42(1). Disponible en: http://scielo.sld.cu/ scielo.php?script=sci_arttext\&pid=S0138-

\section{$600 \times 2016000100011 \& \operatorname{lng}=e s$}

7. Camacaro P. Técnicas e instrumentos de recolección de datos en la investigación cualitativa. Venezuela: Academia Española; 2012

8. Martínez M. El conocimiento: su naturaleza y principales herramientas para su gestión. Encuentros multidisciplinares [Internet] 2010 [consultado 2016 abril 28]; 12(36): 2-17. Disponible en: http://www.encuentrosmultidisciplinares.org/Revistan\%BA36/ MARIA\%20AURORA\%20MARTINEZ\%20REY. pdf

9. Cárdenas-García LJ, Sánchez-Zamora ME, Ramírez-de la Roche OF, et al. F Uso de la anticoncepción de emergencia en un grupo de estudiantes universitarias. Atención Familiar [Internet] 2014 [consultado 2020 junio 17]; 21(3): 90-93. Disponible en: https://www.medigraphic. com/pdfs/atefam/af-2014/af143g.pdf

10. Flores-Pardave R. Nivel de conocimiento sobre la píldora de emergencia y el uso para prevenir el embarazo no deseado en usuarias del Hospital materno infantil Carlos Showing Ferrari 2016. [Tesis]. Universidad de Huánuco; 2017. Disponible en: http://repositorio.udh.edu.pe/bitstream/ handle/123456789/442/T052_22670510_T. pdf? sequence $=3 \&$ isAllowed $=y$

11.Estrella-Cóndor JG, Poma-Mendoza Z. Nivel de conocimiento y uso de la anticoncepción oral de emergencia en un Instituto de Educación no Universitario Palian 2018. [Tesis]. Huancayo: Universidad Peruana Los Andes; 2019. Disponible en: http:// www.repositorio.upla.edu.pe/bitstream/ handle/20.500.12848/1110/TESIS\%20FINAL. pdf?sequence $=1 \&$ is Allowed $=y$

12. Santander-Mamani S. Factores sociodemográficos y conocimiento sobre uso de la píldora anticonceptiva de emergencia en adolescentes de la institución educativa secundaria José María Arguedas de Balsapata, orurillo-2018. [Tesis]. Puno: Universidad Nacional del Altiplano; 2019. Disponible en: http://repositorio.unap.edu.pe/bitstream/handle

UNAP/13350/Santander_Mamani_Sulma. pdf? sequence $=1$ \&isAllowed $=y$ 
13. Villena-Cornejo E. Conocimientos y actitudes sobre la anticoncepción de emergencia hormonal en estudiantes de 4 to y 5 to grado de secundaria de la I.E. Independencia Americana. [Tesis]. Arequipa: Universidad Nacional de San Agustín; 2014. Disponible en: http://repositorio.unsa.edu. pe/bitstream/handle/UNSA/4313/MDvicoea. pdf?sequence=1\&isAllowed=y

Correspondencia:

mvelardec@unjbg.edu.pe
Fecha de recepción: 25/06/2021

Fecha de aceptación: 09/08/2021 